\title{
Vibrational delocalization in ammonia aerosol particles
}

\section{Journal Article}

Author(s):

Jetzki, Martin; Bonnamy, Anthony; Signorell, Ruth (D)

Publication date:

2004

Permanent link:

https://doi.org/10.3929/ethz-b-000429315

Rights / license:

In Copyright - Non-Commercial Use Permitted

Originally published in:

The Journal of Chemical Physics 120(24), https://doi.org/10.1063/1.1752889 


\section{Vibrational delocalization in ammonia aerosol particles}

Cite as: J. Chem. Phys. 120, 11775 (2004); https://doi.org/10.1063/1.1752889

Submitted: 09 February 2004 . Accepted: 30 March 2004 . Published Online: 08 June 2004

Martin Jetzki, Anthony Bonnamy, and Ruth Signorell

ARTICLES YOU MAY BE INTERESTED IN

Infrared spectroscopy of the solid phases of ammonia

The Journal of Chemical Physics 120, 7153 (2004); https://doi.org/10.1063/1.1669380

Cluster Formation in Expanding Supersonic Jets: Effect of Pressure, Temperature, Nozzle Size, and Test Gas

The Journal of Chemical Physics 56, 1793 (1972); https://doi.org/10.1063/1.1677455

Evolution of the vibrational spectrum of ammonia from single molecule to bulk

The Journal of Chemical Physics 128, 134509 (2008); https://doi.org/10.1063/1.2884927
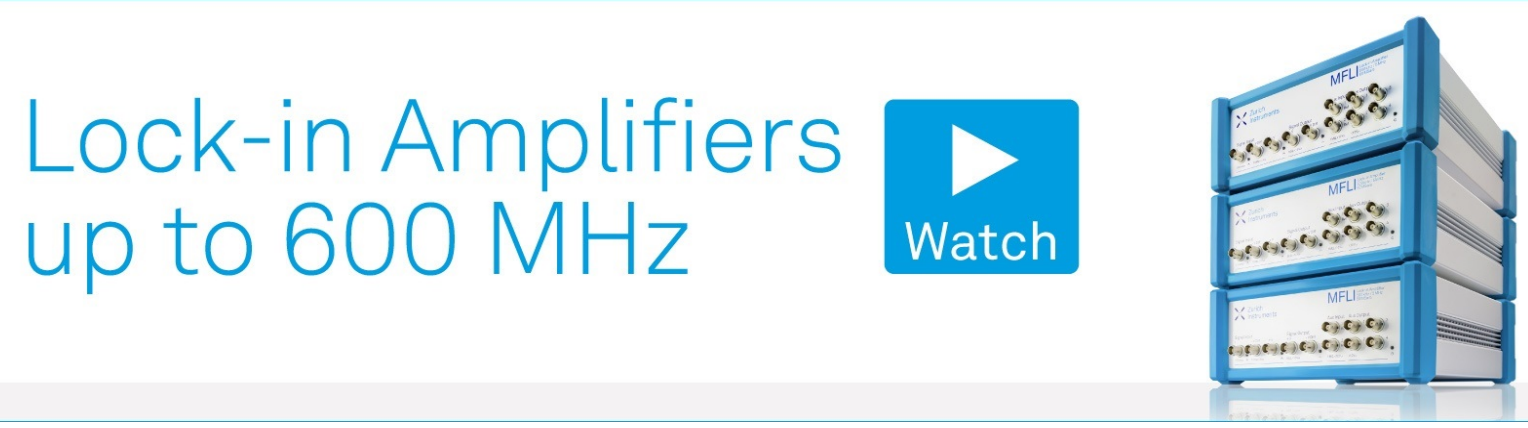

J. Chem. Phys. 120, 11775 (2004); https://doi.org/10.1063/1.1752889

120, 11775

(c) 2004 American Institute of Physics. 


\title{
Vibrational delocalization in ammonia aerosol particles
}

\author{
Martin Jetzki, Anthony Bonnamy, and Ruth Signorell ${ }^{\text {a) }}$ \\ Institut für Physikalische Chemie, Universität Göttingen, Tammannstr. 6, D-37077 Göttingen
}

(Received 9 February 2004; accepted 30 March 2004)

The present contribution investigates shape effects and surface effects in the infrared spectra of pure $\left(\mathrm{NH}_{3}\right)$ and mixed $\left(\mathrm{NH}_{3}-\mathrm{CO}_{2}\right.$ and $\left.\mathrm{NH}_{3}-\mathrm{NH}_{2} \mathrm{D}-\mathrm{NHD}_{2}-\mathrm{ND}_{3}\right)$ ammonia particles with sizes between about 1 and $50 \mathrm{~nm}$. The particles investigated have been generated in a collisional cooling cell as aerosols at temperatures between 20 and $80 \mathrm{~K}$. The contribution reveals that only the combination with a microscopic model leads to a comprehensive understanding of the various features observed in the experimental infrared spectra. As one of the major results, the corresponding exciton model explains why pronounced shape effects observed for pure particles only play a minor role in the case of mixed particles. (C) 2004 American Institute of Physics.

[DOI: $10.1063 / 1.1752889]$

\section{INTRODUCTION}

The combination of collisional cooling and Fourier transform infrared spectroscopy is a powerful tool to investigate the properties of molecular particles with sizes ranging from the micrometer to the subnanometer region (see, for instance, Refs. 1-9 and references therein). The interest in the spectroscopic properties of these huge molecular aggregates arises from their growing relevance in many different fields. They range from medical applications, ${ }^{10}$ to atmospheric processes, ${ }^{11}$ and also to astrophysics. ${ }^{12}$ Intrinsic particle properties such as the particle size, the particle shape, or structural changes in the particle's surface characteristically influence the vibrational dynamics of these aggregates. So far, it is still a challenge to understand such intrinsic particle properties on a microscopic level, that means in terms of inter- and intramolecular interactions.

The present paper focusses on the influence of shape effects on infrared spectra of pure and mixed ammonia particles in the size range between about 10 and $50 \mathrm{~nm}$. Shape effects in pure ammonia particles have previously been investigated by Clapp and Miller in order to clarify their implication for remote sensing of $\mathrm{NH}_{3}$ particles in planetary atmospheres. ${ }^{1}$ Their interpretation of the observed phenomena was based on classical scattering theory. ${ }^{13,14}$ Such calculations, however, do not lead to a microscopic explanation of the underlying mechanisms. Therefore, one goal of the present contribution is to fill this gap by analyzing the experimentally observed shape effects in terms of a molecular model (Sec. IV). In a previous contribution on $\mathrm{CO}_{2}$ particles, ${ }^{9}$ we have already demonstrated the power of this exciton model to explain shape effects in infrared spectra. For instance in the atmosphere, particles are in general composed of different substances. Thus, it is of special interest also to investigate the properties of such mixed particles. In Sec. V, the effect of mixing on shape effects is discussed for the example of isotopically mixed ammonia particles and

\footnotetext{
a) Author to whom correspondence should be addressed. Fax: +49 551 393117. Electronic mail: rsignor@gwdg.de
}

mixed $\mathrm{CO}_{2}-\mathrm{NH}_{3}$ particles. It is demonstrated that mixing has a decisive influence on shape effects.

The paper concludes in Sec. VI with investigations on nanosized ammonia particles. In this size range, i.e., below about $10 \mathrm{~nm}$, the influence of the particle surface is no longer negligible. For the smallest particles, the portion of surface molecules can even dominate over the portion of molecules in the interior of the particle. Because the particle surface in general has not the same structure as the interior this can lead to characteristic surface absorption. Such phenomena have been investigated by Devlin and Buch for the example of small ice particles by combining infrared spectroscopy with extensive calculations. ${ }^{6}$ Here, we report on the first experimental hints that similar phenomena also occur in ammonia particles.

\section{EXPERIMENT AND CALCULATIONS}

The ammonia particles were generated directly from the gas phase in a so-called collisional cooling cell. ${ }^{15,16}$ In cooling cells, particles are formed by injecting a dilute sample gas into a cold bath gas (here Helium at $T>20 \mathrm{~K}$ ). The cooling of the sample gas by the cold bath gas leads to supersaturation of the sample gas, and consequently nucleation and condensation take place. In cooling cells, the particles are generated as aerosols. The particle formation is influenced by the concentration of the sample gas (here typically $100-4000 \mathrm{ppm}$ ), by the pressure of the bath gas (here typically $50-1000 \mathrm{mbar}$ ), and above all by the temperature of the bath gas (here typically $20-80 \mathrm{~K}$ ). The latter strongly influences the size of the particles generated. By cooling the bath gas from liquid nitrogen temperatures down to liquid helium temperatures, ${ }^{8,16}$ aggregates can be formed over a vast size range which extends from some micrometers down to the subnanometer region (Sec. VI). $\mathrm{NH}_{3}(99.98 \%)$ was bought from Messer. To prepare the isotopic mixtures, we have used $\mathrm{ND}_{3}(99 \% \mathrm{D})$ from Cambridge Isotope Laboratories, Inc. The cooling cells used for the present investigations are equipped with White optics and are connected to the infrared spectrometer. This allows to observe even very small particle 

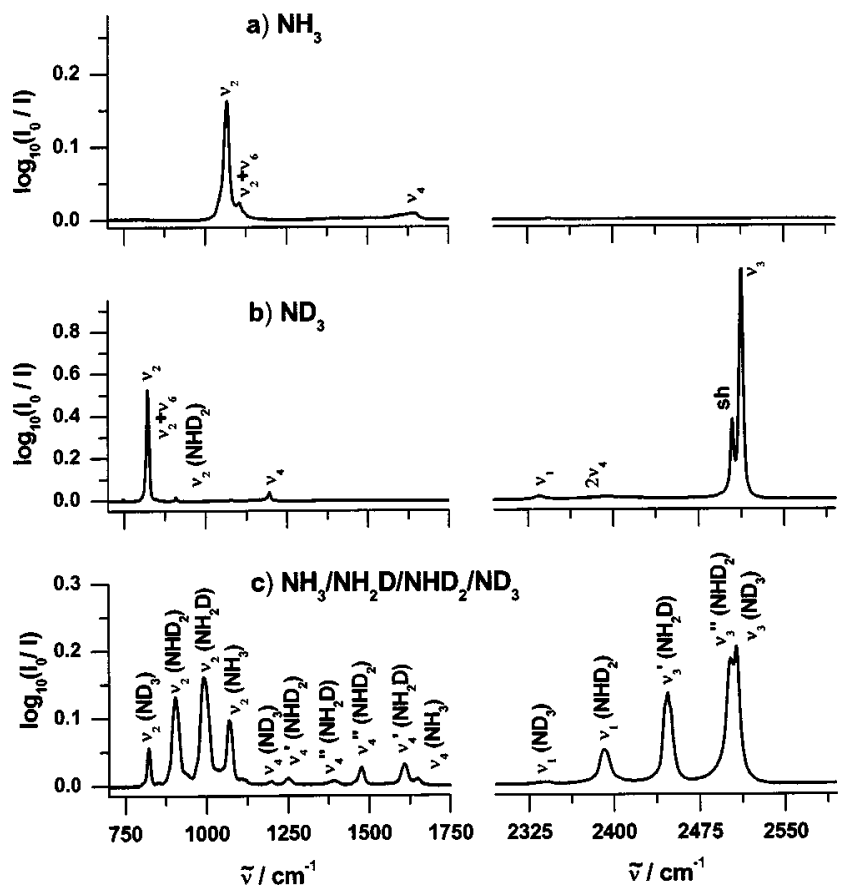

concentrations in the infrared. ${ }^{8,17}$ The vibrational dynamics of the aerosols was studied with a rapid-scan Fourier transform infrared (FTIR) spectrometer (Bruker IFS $66 \mathrm{v} / \mathrm{S}$ ) equipped with a Globar light source, a $\mathrm{KBr}$ beam splitter, and a $\mathrm{HgCdTe}$ detector. The spectral resolution was set to $1 \mathrm{~cm}^{-1}$.

In the present contribution, we use the quantummechanical exciton model ${ }^{18-24}$ to calculate infrared spectra in the region of strong absorption bands. It is a good approximation if the particles consist of equivalent molecules (resonance condition) and if the corresponding molecular transition dipoles are comparatively strong (transition dipoles $>0.1-0.2$ D).$^{23,24}$ In this case, the resonant coupling between the oscillating dipoles of the individual molecules in a particle turns out to be the dominant contribution to the infrared spectra. The corresponding vibrational Hamiltonian has the form:

$$
\hat{H}=\hat{H}_{0}+\hat{H}_{D},
$$

with

$$
\hat{H}_{D}=\sum_{i<j}-\frac{1}{4 \pi \epsilon_{0}} \boldsymbol{\mu}_{i} \cdot \frac{3\left(\boldsymbol{\mu}_{j} \cdot \mathbf{r}_{i j}\right) \cdot \mathbf{r}_{i j}-\left(\mathbf{r}_{i j} \cdot \mathbf{r}_{i j}\right) \cdot \boldsymbol{\mu}_{j}}{r_{i j}^{5}} .
$$

$\hat{H}_{0}$ is the sum over the vibrational Hamiltonians of the uncoupled molecules in a particle. $\hat{H}_{D}$ includes all pairwise dipole-dipole interactions between the molecules in the aggregate. $\mathbf{r}_{i j}$ is the distance between the centers of mass of the molecules labeled $i$ and $j$ and $\boldsymbol{\mu}$ is the dipole moment operator. Up to first-order terms in the vibrational coordinates, the model contains only two parameters: The transition wave number of the uncoupled molecule $\widetilde{\nu}_{M}$ and the transition dipole moment of the uncoupled molecule $\delta \mu=\langle 0|\mu| 1\rangle$. Both quantities can be extracted from gas-phase measurements or from $a b$ initio calculations on smaller clusters (Secs. IV and V). The diagonalization of $\hat{H}$ leads to the vibrational eigen-

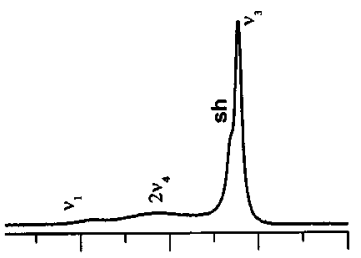

FIG. 1. Survey spectra of (a) pure $\mathrm{NH}_{3}$ particles, (b) pure $\mathrm{ND}_{3}$ particles, and (c) a 1:3:3:1 mixture of $\mathrm{NH}_{3}$, $\mathrm{NH}_{2} \mathrm{D}, \mathrm{NHD}_{2}$, and $\mathrm{ND}_{3}$. The $\mathrm{ND}_{3}$ spectrum in panel (b) shows only very small contributions from the other isotopomers. The wavenumbers of the fundamental transitions are listed in Table I. function and the vibrational eigenvalues of the particles. From these quantities, the infrared spectra of the particles were calculated. $^{23}$

\section{SURVEY SPECTRA}

Figure 1 shows survey spectra of ammonia ice aerosols between 700 and $3500 \mathrm{~cm}^{-1}$ for different isotopomers. All particles were generated in a collisional cooling cell at a bath gas temperature of $78 \mathrm{~K}$. The infrared spectra obtained for $\mathrm{NH}_{3}$ particles and $\mathrm{ND}_{3}$ particles are depicted in Figs. 1(a) and 1(b), respectively. Figure 1(c) contains the particle spectrum of a mixture of $\mathrm{NH}_{3}, \mathrm{NH}_{2} \mathrm{D}, \mathrm{NHD}_{2}$, and $\mathrm{ND}_{3}$. This isotopically mixed particles were formed from a 1:1 mixture of $\mathrm{NH}_{3}$ and $\mathrm{ND}_{3}$ gas which had been equilibrated before use for several hours at $298 \mathrm{~K}$. The equilibrium gas, and consequently the particles, consist of a 1:3:3:1 mixture of $\mathrm{NH}_{3}$, $\mathrm{NH}_{2} \mathrm{D}, \mathrm{NHD}_{2}$, and $\mathrm{ND}_{3}$. In this context it is important to note that the integrated band strengths do not only scale with the portion of the corresponding isotopomers but also with the transition wavenumber and with the square of their transition dipole moments which differ for different isotopomers. $^{25,26}$

The particle spectra in Fig. 1 do not show any contribution from elastic scattering, i.e., a dispersion shape of the absorption peaks or a raising of the baseline towards higher frequencies. ${ }^{13,27}$ As described in more detail in Ref. 15, this observation can be used to estimate the size of the aerosol particles. Briefly, this is done by employing Mie calculations using the refractive index data for $\mathrm{NH}_{3}$ from Refs. 28 and 29. As a result of this calculations, we obtain an upper limit for the mean particle radius of about $50 \mathrm{~nm}$. The lower limit lies around $10 \mathrm{~nm}$. This value results from the fact that between about 10 and $50 \mathrm{~nm}$ the band structures and the relative band intensities are to a good approximation independent of the 
TABLE I. Fundamental transitions in $\mathrm{cm}^{-1}$ of $\mathrm{NH}_{3}, \mathrm{ND}_{3}, \mathrm{NH}_{2} \mathrm{D}$, and $\mathrm{NHD}_{2}$ in small crystalline particles (this work and Ref. 40) and in crystalline layers (Refs. 30-32). The values for $\mathrm{NH}_{3}$ and $\mathrm{ND}_{3}$ are obtained from isotopically pure samples [Figs. 1(a) and 1(b)] those for $\mathrm{NH}_{2} \mathrm{D}$ and $\mathrm{NHD}_{2}$ are taken from 1:3:3:1 mixture of $\mathrm{NH}_{3}, \mathrm{NH}_{2} \mathrm{D}, \mathrm{NHD}_{2}$, and $\mathrm{ND}_{3}$ [Fig. 1(c)]. The notations of the vibrational bands follows Ref. 32 .

\begin{tabular}{|c|c|c|c|c|c|}
\hline Band & Ref. & $\mathrm{NH}_{3}$ & $\mathrm{ND}_{3}$ & $\mathrm{NH}_{2} \mathrm{D}(1: 3: 3: 1)^{\mathrm{a}}$ & $\mathrm{NHD}_{2}(1: 3: 3: 1)^{2}$ \\
\hline \multirow[t]{5}{*}{$\nu_{1}$} & this work (Fig. 1) & 3216 & 2335 & 3300 & 2391 \\
\hline & 32 & 3213 & 2335 & 3300 & 2391 \\
\hline & 30 and 31 & 3223 & 2318 & 3307 & 2389 \\
\hline & this work (Fig. 8) ) $^{\mathrm{c}, \mathrm{d}}$ & 3209 & & & \\
\hline & $40^{\mathrm{c}}$ & 3204 & & & \\
\hline \multirow[t]{7}{*}{$\nu_{2}$} & this work (Fig. 1) & 1066 & 822 & & \\
\hline & 32 & 1059 & 814 & & \\
\hline & 30 & 1060 & 815 & & \\
\hline & this work [Fig. 1(c)] & $1068(1: 3: 3: 1)^{\mathrm{a}}$ & $822(1: 3: 3: 1)^{\mathrm{a}}$ & 993 & 904 \\
\hline & 32 & $1068(1: 3: 3: 1)^{\mathrm{a}}$ & $820(1: 3: 3: 1)^{\mathrm{a}}$ & 988 & 901 \\
\hline & 31 & $1069(1: 3: 3: 1)^{\mathrm{a}}$ & $824(1: 3: 3: 1)^{\mathrm{a}}$ & 993 & 905 \\
\hline & this work (Fig. 8) $)^{\mathrm{c}, \mathrm{d}}$ & 1069 & & & \\
\hline \multirow{5}{*}{$\nu_{3}$} & this work (Fig. 1) & 3378 & 2513 & & \\
\hline & 32 & 3376 & 2511 & & \\
\hline & 30 & 3378 & $2500^{\mathrm{e}}$ & & \\
\hline & this work (Fig. 8) ) $^{\mathrm{cd}}$ & 3378 & & & \\
\hline & $40^{\mathrm{c}}$ & 3370 & & & \\
\hline \multirow[t]{2}{*}{$\nu_{3}(\mathrm{sh})^{\mathrm{b}}$} & this work (Fig. 1) & 3369 & 2504 & & \\
\hline & 32 & 3369 & 2504 & & \\
\hline \multirow[t]{3}{*}{$\nu_{3}^{\prime}$} & this work (Fig. 1) & & & 2447 & 3329 \\
\hline & 32 & & & 2447 & 3329 \\
\hline & 31 & & & 2444 & 3338 \\
\hline \multirow[t]{3}{*}{$\nu_{3}^{\prime \prime}$} & this work (Fig. 1) & & & 3361 & 2502 \\
\hline & 32 & & & 3360 & 2501 \\
\hline & 31 & & & 3378 & 2500 \\
\hline \multirow[t]{3}{*}{$\nu_{4}$} & this work (Fig. 1) & 1644 & 1196 & & \\
\hline & 32 & 1648 & 1196 & & \\
\hline & 30 & 1646 & 1196 & & \\
\hline \multirow[t]{3}{*}{$\nu_{4}^{\prime}$} & this work (Fig. 1) & & & 1608 & 1252 \\
\hline & 32 & & & 1609 & 1253 \\
\hline & 31 & & & 1609 & 1255 \\
\hline \multirow[t]{3}{*}{$\nu_{4}^{\prime \prime}$} & this work (Fig. 1) & & & 1392 & 1477 \\
\hline & 32 & & & 1393 & 1476 \\
\hline & 31 & & & 1393 & 1476 \\
\hline \multirow[t]{5}{*}{$2 \nu_{4}$} & this work (Fig. 1) & 3285 & 2392 & & \\
\hline & 32 & 3291 & 2395 & & \\
\hline & 30 & 3297 & 2390 & & \\
\hline & this work (Fig. 8) $)^{\mathrm{c}, \mathrm{d}}$ & 3287 & & & \\
\hline & $40^{\mathrm{c}}$ & 3294 & & & \\
\hline \multirow[t]{2}{*}{$\nu_{2}+\nu_{6}$} & this work (Fig. 1) & 1105 & 849 & & \\
\hline & 30 & 1100 & 849 & & \\
\hline
\end{tabular}

${ }^{a}$ Values obtained from 1:3:3:1 mixture of $\mathrm{NH}_{3}, \mathrm{NH}_{2} \mathrm{D}, \mathrm{NHD}_{2}$, and $\mathrm{ND}_{3}$.

$\mathrm{b}_{\mathrm{sh}}=$ shoulder.

${ }^{\mathrm{c}}$ Nanosized particles.

${ }^{\mathrm{d}}$ Values from the $21 \mathrm{~K}$ spectrum depicted in Fig. 8

${ }^{\mathrm{e}}$ The difference of this value arises most probably from contributions of the isotopomer $\mathrm{NHD}_{2}$ in the $\mathrm{ND}_{3}$ spectrum of Ref. $31\left[\nu_{3}^{\prime \prime}\left(\mathrm{NHD}_{2}\right)\right.$ in Fig. 1]

particles size. ${ }^{13,27}$ We have tested the stability of the spectra in this size range by recording particle spectra for sample gas mixtures with different $\mathrm{NH}_{3}$ concentrations.

From a comparison with earlier infrared and Raman studies of solid ammonia and of ammonia aerosols, ${ }^{1,28-33}$ we conclude that our aerosol particles are crystalline and have the cubic $\mathrm{P} 2{ }_{1} 3$ structure of the bulk. ${ }^{34-38}$ This conclusion is also supported by an electron diffraction study of large ammonia clusters produced in a Laval nozzle ${ }^{39}$ which shows that frozen crystals of the cubic phase are formed within a dozen microseconds.

An assignment of the observed bands is given in Table I.
The notation follows Ref. 32. Note that the correct site symmetry in the pure $\mathrm{NH}_{3}$ and in the pure $\mathrm{ND}_{3}$ particles [Figs. 1(a) and 1(b), respectively] is $\mathrm{C}_{3}$, but that $\mathrm{C}_{3 v}$ still approximates the situation (no inversion motion in the condensed phase). In the mixed particles [Fig. 1(c)], there is no symmetry. But for $\mathrm{NH}_{2} \mathrm{D}$ and $\mathrm{NHD}_{2}$, the group $\mathrm{C}_{s}$ again approximates the symmetry. Table I compares the values of the transition wavenumbers measured in the present contribution with corresponding data for crystalline ammonia layers. ${ }^{30-32}$ The present values represent peak maxima values. In case of the literature values, however, it is not clear what exactly they represent. For most of the bands listed in Table I 
(especially for the broad bands and for the weak bands), this probably explains the deviations between the data for thin films and the present data for particles. In other words, this also means that the influence of particle properties (e.g., shape effects) is rather minor for most of the vibrational bands. The only exception is the bending vibration $\nu_{2}$ in the pure $\mathrm{NH}_{3}$ and the pure $\mathrm{ND}_{3}$ particles [Figs. 1(a) and 1(b)]. Table I reveals that for the pure particles this band lies systematically higher by $6-8 \mathrm{~cm}^{-1}$ compared with thin layers. ${ }^{30,32}$ This effects has also been observed in earlier particle spectra of ammonia aerosols. ${ }^{1}$ As will be further discussed in the next section, this difference can be traced back to the influence of resonant transition dipole-transition dipole coupling between the equivalent molecules in a particle. We have demonstrated earlier, ${ }^{23,27}$ that this interaction is sensitive to the shape of the sample (here small spheres compared with thin layers). Due to the increased distance between equivalent isotopomers in the isotopically mixed particles in Fig. 1(c), the resonant transition dipoletransition dipole coupling is weakened in these mixed particles [see Eq. (2)]. Consequently the shape-sensitivity disappears as do the differences in the transition wavenumber for $\nu_{2}$ between particles and layers. ${ }^{31,32}$ This can be seen in Table I from the values of $\nu_{2}$ for the isotopomers $\mathrm{NH}_{3}$ and $\mathrm{ND}_{3}$ in the mixed samples [labeled $(1: 3: 3: 1)$ in columns 3 and 4] and for the isotopomers $\mathrm{NH}_{2} \mathrm{D}$ and $\mathrm{NHD}_{2}$ in the mixed samples (columns 5 and 6) for which no systematic deviation between particles and layers can be found. The aim of the present contribution is further to investigate this special behavior of $\nu_{2}$ and above all to give for the first time a microscopic explanation for that (Secs. IV and V).

Table I also lists transition wavenumbers for nanosized particles with sizes clearly below $5 \mathrm{~nm}$. One of these data sets is taken from Ref. 40 and the other corresponds to the maximum values obtained from the $21 \mathrm{~K}$ spectrum of Fig. 8 in Sec. VI. Due to the different particle sizes, these data should not be compared directly with the other data in Fig. 1 which are for particles with radii above $10 \mathrm{~nm}$. The reason lies in the influence of the particle's surface in the case of nanosized particles. This aspect will be briefly discussed in Sec. VI.

\section{SHAPE EFFECTS IN PURE PARTICLES}

As mentioned in the previous section, the $\nu_{2}$ band in ammonia particles is more sensitive to the shape of the sample than all other vibrational bands in the mid-infrared region. This shape-dependence explains the differences between particle spectra and spectra of thin layers and also the differences between spectra for different ammonia aerosols as reported by Clapp and Miller. ${ }^{1}$ What is still missing, however, is a microscopic explanation of this phenomenon. To clarify this point further, we have investigated pure ammonia particles as well as mixed particles (following section) using a collisional cooling cell for the particle formation. Previous studies with collisional cooling cells $s^{1,2,5,9,15,41}$ have shown that the shape of the particles mainly depend on the bath gas pressure in the cooling cell and on the time after the particle formation sets in: Particles with predominantly globular shape are formed at high bath gas pressures and immediately
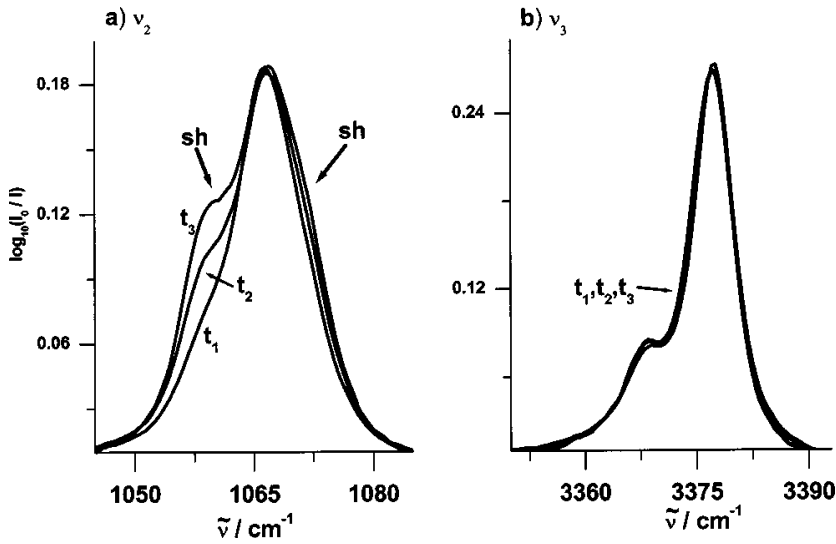

FIG. 2. Infrared spectra of pure $\mathrm{NH}_{3}$ particles in the region of (a) the umbrella vibration $\nu_{2}$ and (b) the degenerate NH-stretching vibration $\nu_{3}$. Each trace shows spectra recorded at different times after the particle formation sets in: $t_{1}=10 \mathrm{~s}, t_{2}=230 \mathrm{~s}$, and $t_{3}=884 \mathrm{~s}$. $\nu_{2}$ forms two shoulders (sh) with increasing time. $\nu_{3}$ is time independent. The sample gas concentration amounted to $1600 \mathrm{ppm} \mathrm{NH}_{3}$ in $\mathrm{He}$.

after the particle formation. Particles with mostly elongated shape are formed at low bath gas pressures and at longer time after the particle formation sets in. Examples for $\mathrm{NH}_{3}$ spectra recorded at different experimental conditions are depicted in Fig. 2. Figure 2(a) shows three particle spectra in the region of the umbrella vibration $\nu_{2}$ which were recorded at different times after the particle formation $\left(t_{1}=10 \mathrm{~s}, t_{2}\right.$ $=230 \mathrm{~s}$, and $t_{3}=884 \mathrm{~s}$ ). Figure 2(b) shows the same but for the degenerate $\mathrm{NH}$-stretching mode $\nu_{3}$. In the condensed phase, this band is split into two components as can be seen in Fig. 2(b). ${ }^{30-32}$ Though $\nu_{2}$ and $\nu_{3}$ are of comparable strength [Figs. 1(a) and 2] they obviously reveal a very different behavior: While $\nu_{3}$ remains stable with increasing time, $\nu_{2}$ forms two shoulders, a marked shoulder on the lowfrequency side of the main peak, and a weaker one on the high frequency side. The same behavior is found for decreasing bath gas pressure in the cell and also in the case of pure $\mathrm{ND}_{3}$ particles. Clapp and Miller have attributed this special behavior of $\nu_{2}$ to a change in the particle's shape. ${ }^{1}$ They suggested that the time-dependence could be traced back to aggregation of smaller particles into chains, i.e., to the formation of elongated particles. The formation of a variety of particle shapes for large ammonia ice particles had earlier been observed by Pope et al. ${ }^{42}$ In previous investigations on $\mathrm{CO}_{2}$ and $\mathrm{N}_{2} \mathrm{O}$ particles, ${ }^{8,9}$ we have also found similar shape effects. From our experimental data, however, we could not clearly decide whether the elongated particles were formed by dendritic (needlelike) growth or by agglomeration of smaller particles.

The understanding of such shape effects requires the modeling of the spectra. Clapp and Miller $^{1}$ have compared their observed spectra with calculated spectra for elongated particles using the discrete dipole approximation (DDA see also Refs. 14 and 43). The comparison of their experimental data with the simulations leads to a good qualitative agreement which at least supports the occurrence of shape effects. This classical model, however, does not explain the microscopic mechanisms which lie behind such phenomena. The DDA approximation is based on macroscopic quantities as 
input data such as refractive index data. Often, these data are obtained from infrared spectra of thin films. The aim of the present investigation is to give a microscopic explanation, i.e., to calculate spectra directly from molecular properties.

In previous studies on $\mathrm{CO}_{2}$ and $\mathrm{N}_{2} \mathrm{O}$ aerosol particles, ${ }^{9,23,27}$ we have shown that shape effects in infrared spectra can be traced back to resonant couplings between the transition dipole moments of the individual molecules in a particle. The corresponding interaction follows Eq. (2) which shows that the coupling is largely governed by molecular properties: The transition dipole moments $\delta \mu_{M}$ of the individual molecules and their transition wavenumbers $\widetilde{\nu}_{M}$. The coupling scales as $1 / r_{i j}^{3}$ with the distance $r_{i j}$ between the molecules. Shape effects are important if this resonant interaction is dominant. In a recent contribution, ${ }^{24}$ this led us to the formulation of the following propensity rules for the occurrence of strong shape effects in infrared spectra of molecular particles: (i) The particles consist of molecules with strong molecular transition dipoles $\left(\delta \mu_{M}>0.1-0.2 \mathrm{D}\right)$. (ii) The particles only contain spectrally equivalent molecules (resonance condition). (iii) The equivalent molecules are separated by small distances $\left(r_{i j}<5-7 \AA\right)$. In the case of the $\nu_{2}$ band in pure $\mathrm{NH}_{3}$ particles all three conditions are fulfilled: (i) The molecular transition dipole amounts to $\delta \mu_{M}$ $\sim 0.23$ D. ${ }^{25,26,44,45}$ (ii) In the cubic ammonia crystal, ${ }^{34-38}$ all molecules are equivalent and are in "equivalent" surroundings. (iii) The distance between neighboring molecules amounts to $\sim 3.3-3.9 \AA$. Consequently, the occurrence of strong shape effects for the umbrella band is in full agreement with the predictions of our model. In addition, this model explains why in case of the $\nu_{3}$ band no shape effects are observed [Fig. 2(b)]. The reason lies in the small molecular transition dipole moment which now amounts to $\delta \mu_{M}$ $\sim 0.02 \mathrm{D}$. Such a small value only leads to a very weak transition dipole interaction which is in fact negligible compared with other interactions. As a consequence, the NHstretching band exhibits no shape effects. These considerations illustrate how shape effects are influenced by molecular properties.

With the exciton model at hand, we can now calculate shape effects in infrared spectra directly from molecular properties. For the $\nu_{2}$ band, two examples for calculated spectra (upper traces) are depicted in Fig. 3 together with the corresponding experimental infrared spectra (lower traces). The experimental spectra in Figs. 3(a) and 3(b) correspond to the spectra in Fig. 2(a) which have been recorded $10 \mathrm{~s}\left(t_{1}\right)$ and $884 \mathrm{~s}\left(t_{3}\right)$, respectively, after the particle formation sets in. The two calculated spectra represent two different mixtures of globular and elongated particles. In Fig. 3(a), the ratio of globular to elongated particles amounts to 1:1.7. The spectral features in this picture are mainly governed by the globular particles. For simplicity, we thus identify the corresponding particles with globular particles as implied in Fig. 3(a). In Fig. 3(b), the ratio of globular to elongated particles amounts to 1:6. These spectra are characteristically influenced by the elongated particles which lead to the marked shoulders. Therefore, we identify these particles for simplicity with elongated particles. In both cases, the globular particles are simulated by a crystalline sphere with a diameter of
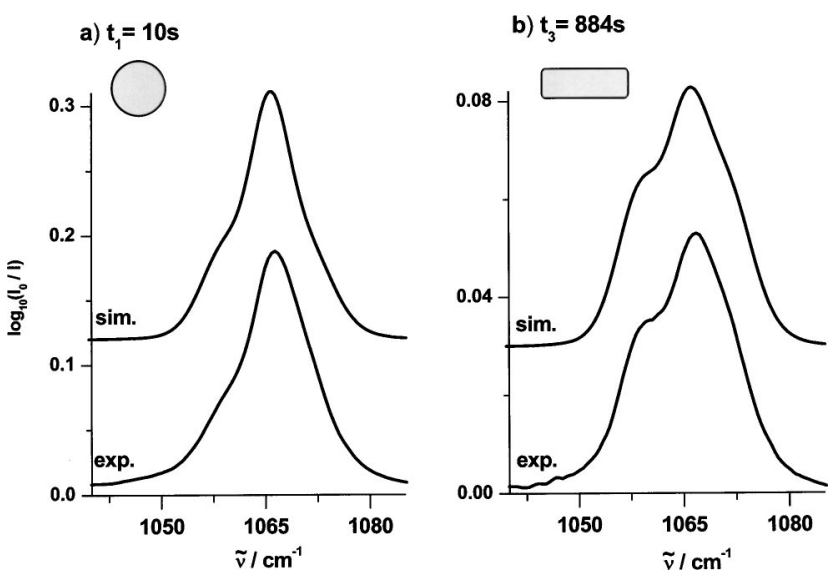

FIG. 3. Infrared spectra of pure $\mathrm{NH}_{3}$ particles in the region of the umbrella vibration $\nu_{2}$ (a) $10 \mathrm{~s}$ after particle formation sets in and (b) $884 \mathrm{~s}$ after particle formation sets in. Lower traces: experimental spectra. Upper traces: Exciton calculations. Panel (a) corresponds to a 1:1.7 mixture of globular and elongated particles. In panel (b), the ration of globular to elongated particles amounts to $1: 6$.

about $8 \mathrm{~nm}$. The elongated particle is a crystalline rectangular parallelepiped with an axis ratio of 4:1:1 $(32 \times 8 \times 8$ unit cells). We have tested the convergence of the spectra with size by increasing the number of molecules per particle by up to a factor of 2. In accordance with the results of Sec. III, all particles are build up from the cubic unit cell of the crystalline bulk. ${ }^{36,38}$ As an example, Fig. 4(a) shows a small crystalline sphere which consists of $\sim 2000$ molecules (radius 2.5 $\mathrm{nm}$ ). The molecular properties enter the exciton calculations through the transition wave number $\widetilde{\nu}_{M}$ and the transition dipole moment $\delta \mu_{M}$ (see Sec. II). $\widetilde{\nu}_{M}$ and $\delta \mu_{M}$ correspond to the values of the uncoupled local molecular oscillators in the solid particles. In the case of crystalline $\mathrm{CO}_{2}$ particles, we have found that $\tilde{\nu}_{M}$ and $\delta \mu_{M}$ approximately equal the corresponding gas-phase values of $\mathrm{CO}_{2} \cdot{ }^{9,23,46}$ This, however, is no longer the case for crystalline $\mathrm{NH}_{3}$ particles. Here, the formation of strong intermolecular hydrogen bonds lifts the symmetry of the inversion potential and drastically increases the barrier to inversion. This is accompanied by a marked increase of the transition wavenumber for $\nu_{2}$ in the particles $\left(1066 \mathrm{~cm}^{-1}\right.$ see Table I) compared with the isolated gasphase molecule $\left(950 \mathrm{~cm}^{-1}\right.$ see Ref. 47$)$. The transition wavenumber in the particles lies now close to the value of the crystalline bulk $\left(1060 \mathrm{~cm}^{-1}\right.$ see Table I). In contrast to the value for the particles, the bulk value is not shifted due to size or shape effects. Thus, we have chosen this bulk value as input value for $\widetilde{\nu}_{M}$ for the exciton calculations. The transition dipole moment $\delta \mu_{M}$ is only affected to a minor extent by the aggregation. Therefore, we have set $\delta \mu_{M}=0.21 \mathrm{D}$ which lies within the expected uncertainty of the corresponding experimental gas-phase values. ${ }^{25,26,44,45}$ Equation (3) summarizes the input parameters used for the exciton calculations shown in Fig. 3

$$
\widetilde{\nu}_{M}\left(\mathrm{NH}_{3}\right)=1060 \mathrm{~cm}^{-1} \text { and } \delta \mu_{M}\left(\mathrm{NH}_{3}\right)=0.21 \mathrm{D} \text {. }
$$

We have tried to confirm by ab initio calculations ${ }^{48}$ that the observed shift of $\tilde{\nu}_{M}$ in the condensed phase compared with the gas phase mainly arises from the formation of 
a)

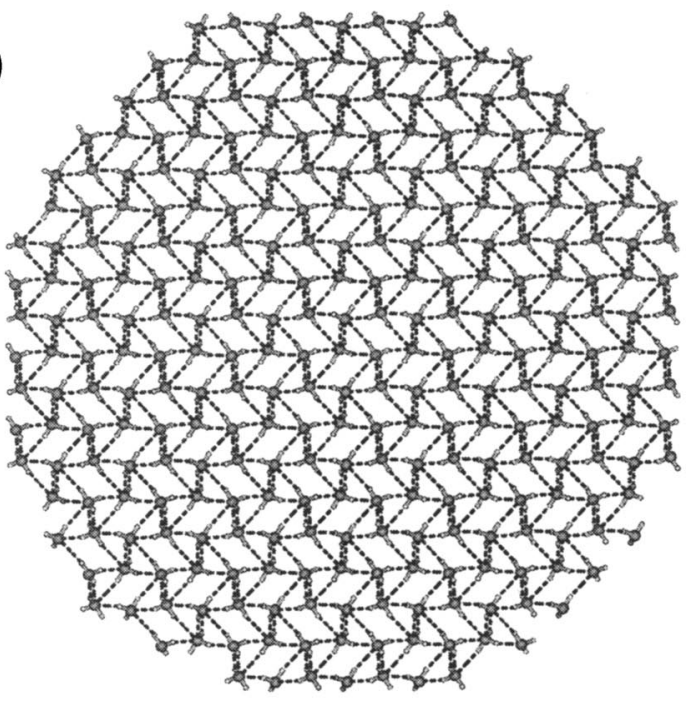

b)

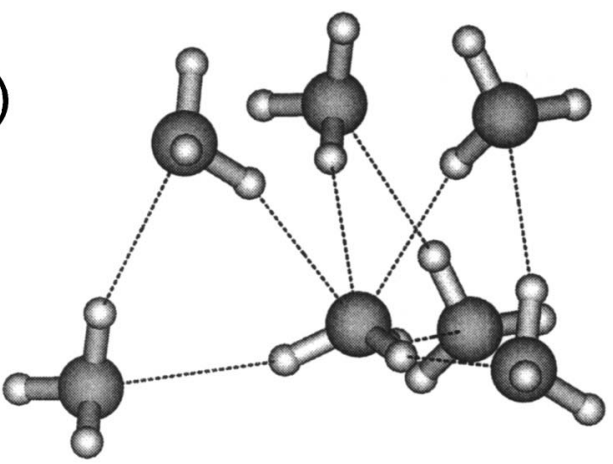

c)

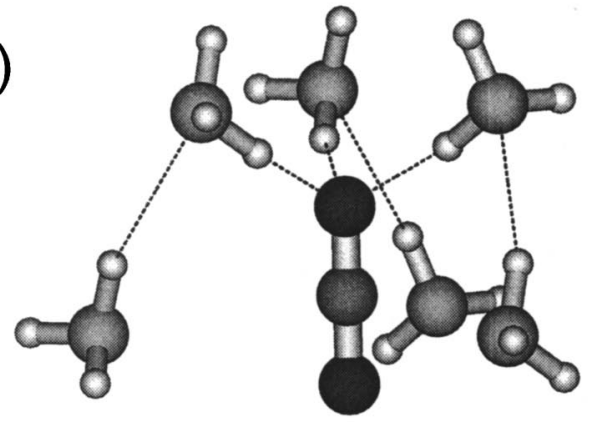

FIG. 4. (a) Small crystalline sphere which contains $\sim 2000$ ammonia molecules (radius $2.5 \mathrm{~nm}$ ). (b) Small $\mathrm{NH}_{3}$ cluster. The central $\mathrm{NH}_{3}$ molecule is surrounded by the six nearest neighbors as in the crystal (Refs. 36 and 38). (c) Small $\mathrm{NH}_{3}-\mathrm{CO}_{2}$ cluster. The central $\mathrm{CO}_{2}$ molecule is surrounded by six $\mathrm{NH}_{3}$ molecules.

hydrogen bonds. For that, the influence of hydrogen bonds is considered for a $\mathrm{NH}_{3}$ molecule which is surrounded by the first shell of nearest neighbors (six molecules) as in the crystal. This small cluster is depicted in Fig. 4(b). The geometry of this cluster is assumed to be the same as in the crystal. ${ }^{36,38}$ Note that due to the different surrounding of the central molecule and the surrounding molecules there is no exciton coupling between the central molecule and the other molecules in this small cluster. This is also confirmed by calculations for which the surrounding molecules were isotopomers of
TABLE II. $\tilde{\nu}_{\text {cluster }}-\widetilde{\nu}_{\text {molecule }}$ represents the calculated difference in the transition wavenumber between a molecule surrounded by six other molecules $\left(\tilde{\nu}_{\text {cluster }}\right)$ as depicted in Figs. 4(b) and 4(c) and an isolated molecule $\left(\tilde{\nu}_{\text {molecule }}\right)$. The second column contains the values for the umbrella vibration of $\mathrm{NH}_{3}$ [Fig. 4(b)]. The third column lists the values for the antisymmetric stretching vibration of $\mathrm{CO}_{2}$ [Fig. 4(c)].

\begin{tabular}{lcc}
\hline \hline & $\begin{array}{c}\mathrm{NH}_{3} \text { in }\left(\mathrm{NH}_{3}\right)_{6} \\
{[\text { see Fig. 4(b)] }}\end{array}$ & $\begin{array}{c}\mathrm{CO}_{2} \text { in }\left(\mathrm{NH}_{3}\right)_{6} \\
{[\text { see Fig. 4(c) }]}\end{array}$ \\
Method (Ref. 48) & $\tilde{\nu}_{\text {cluster }}-\widetilde{\nu}_{\text {molecule }} / \mathrm{cm}^{-1}$ & $\tilde{\nu}_{\text {cluster }}-\widetilde{\nu}_{\text {molecule }} / \mathrm{cm}^{-1}$ \\
\hline B3LYP/6-31+G* & 172 & 4 \\
B3LYP/6-31++G* & 165 & 4 \\
B3LYP/6-31++G** & 169 & 3 \\
B3LYP/aug-cc-pvdz & 191 & -3 \\
B3LYP/aug-cc-pvtz & 160 & -1 \\
MP2/6-31+G* & 103 & 9 \\
MP2/6-31++G* & 137 & 9 \\
MP2/6-31++G* & 127 & 4 \\
MP2/aug-cc-pvdz & 182 & $9.0^{\mathrm{b}}$ \\
Experiment & $110^{\mathrm{a}}$ & 9 \\
\hline
\end{tabular}

${ }^{\text {a }}$ This value corresponds to the difference between the value for the bulk (Ref. 30) and the value for the gas phase (Ref. 47).

${ }^{\mathrm{b}}$ This value corresponds to the difference between the value used for the exciton calculation in Eq. (4) and the value for the gas phase (Ref. 46).

ammonia. Therefore, the transition wavenumber of the central molecule $\tilde{\nu}_{\text {cluster }}$ gives an idea of the influence of the formation of hydrogen bonds when it is compared with the transition wavenumber of an isolated ammonia molecule $\widetilde{\nu}_{\text {molecule }}$. The calculated shifts $\widetilde{\nu}_{\text {cluster }}-\widetilde{\nu}_{\text {molecule }}$ are listed for different levels of theory in the second column of Table II. They show that it is plausible to trace back the experimentally observed shift of $110 \mathrm{~cm}^{-1}$ to the formation of hydrogen bonds.

The above results clearly confirm the earlier explanation of Clapp and Miller ${ }^{1}$ that different particle shapes are responsible for the dependence of the band structure of the umbrella vibration on the experimental conditions. In contrast to the continuum model used by Clapp and Miller, our quantummechanical exciton model for the first time provides a microscopic explanation for the observed shape effects. On the one hand, this results in a much better agreement between calculated and experimental data (Fig. 3). But above all, the microscopic model reveals the resonant transition dipoletransition dipole coupling between individual molecules in a particle as the dominant mechanism behind shape effects. This interaction lifts the degeneracy between the uncoupled resonant states and leads to eigenfunctions that are delocalized over the whole particle. This delocalization explains the distinct shape-dependence.

\section{SHAPE EFFECTS IN MIXED PARTICLES}

In the previous section, we have considered shape effects in infrared spectra of pure $\mathrm{NH}_{3}$ particles. In the atmosphere or in interstellar clouds, however, particles consist in general of different substances. Therefore, it is of special interest to know the spectroscopic properties of such mixed particles. The discussions in Secs. II and IV imply that mixing also has an influence on shape effects. To clarify this point, the present section considers shape effects in the infrared spectra of mixed ammonia particles. 

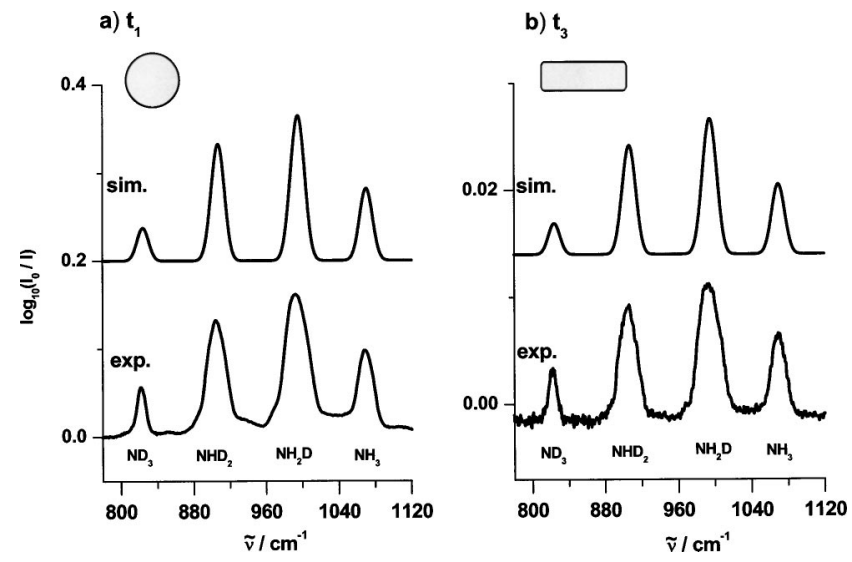

FIG. 5. Infrared spectra in the region of the umbrella vibration $\nu_{2}$ of isotopically mixed particles that consist of a 1:3:3:1 mixture of $\mathrm{NH}_{3}, \mathrm{NH}_{2} \mathrm{D}$, $\mathrm{NHD}_{2}$, and $\mathrm{ND}_{3}$. Lower traces: Experimental spectra. (a) Spectrum measured directly after the particle formation sets in. (b) Spectrum measured after several minutes (see caption Fig. 3). Upper traces: Exciton calculations. Panel (a) corresponds to a 1:1.7 mixture of globular and elongated particles. In panel (b), the ration of globular to elongated particles amounts to $1: 6$. For the exciton calculations, we have used the values of Refs. 25 and 26 for the transition dipole moments $\delta \mu_{M}$ of the different isotopomers. The transition frequencies $\widetilde{\nu}_{M}$ correspond to the data for the thin layers $(1: 3: 3: 1$ mixture) listed in Table I.

\section{A. Isotopically mixed particles}

One of the simplest mixtures is a mixture of different isotopomers. Most of the particle properties as for instance the crystal structure or the shape of the particles remain nearly unaffected by isotope substitution. There is, however, one exception: The transition wavenumber $\widetilde{\nu}_{M}$ (see Sec. II). It can differ substantially for different isotopomers. As a consequence, the resonance condition for exciton coupling is partially lifted in isotopically mixed particles. For large differences in $\widetilde{\nu}_{M}$, the coupling between molecules of different isotopomers is in fact negligible. The molecules of the same isotopomer still couple but the mean distance between them is increased in a statistically mixed particle compared with a pure particle. Due to the $1 / r_{i j}^{3}$ term in Eq. (2) this reduces the effect of exciton coupling. In the infrared spectra this should manifest itself at least in a partial loss of the characteristic band structure which is observed for different particle shapes for pure ammonia particles [seen Figs. 2(a) and 3].

That this really happens is illustrated in Fig. 5 for particles consisting of a 1:3:3:1 mixture of $\mathrm{NH}_{3}, \mathrm{NH}_{2} \mathrm{D}$, $\mathrm{NHD}_{2}$, and $\mathrm{ND}_{3}$. These particles have been generated under the same experimental conditions as the pure $\mathrm{NH}_{3}$ particles whose spectra are depicted in Fig. 3. Therefore, the spectrum in Fig. 5(a) corresponds to a 1:1.7 mixture of globular and elongated particles and the spectrum in Fig. 5(b) corresponds to a 1:6 mixture of globular and elongated particles (see text to Fig. 3). The comparison of the experimental band structures of the umbrella motion of $\mathrm{NH}_{3}$ in Fig. 5 with those of Fig. 3 nicely shows that the information about the particle shape is really lost in the spectra of the isotopically mixed particles. While the band shape of $\nu_{2}$ for the pure $\mathrm{NH}_{3}$ particles in Fig. 3 obviously differs for different particle shapes [Fig. 3(a) compared with Fig. 3(b)] the band shape of $\nu_{2}$ for the $\mathrm{NH}_{3}$ absorption in the mixed particles in Fig. 5 does not

$$
15 \% \mathrm{CO}_{2} / 85 \% \mathrm{NH}_{3}: \quad 100 \% \mathrm{CO}_{2}:
$$
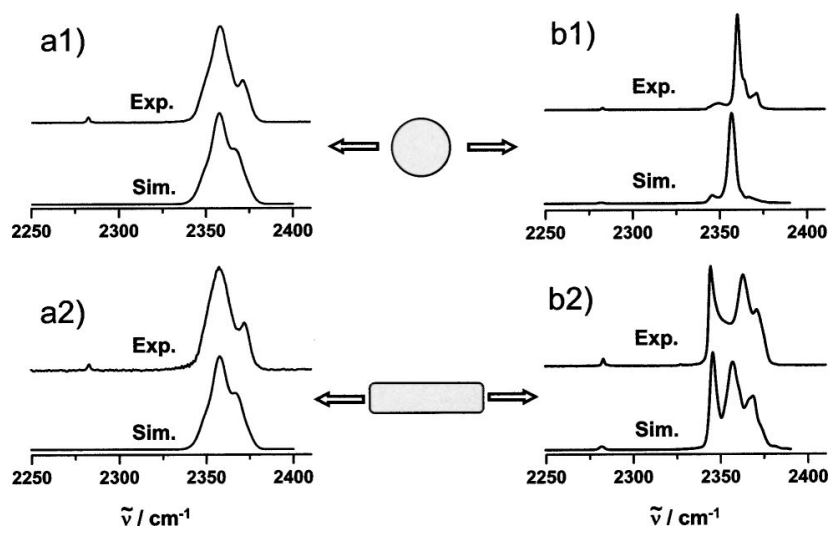

FIG. 6. Infrared spectra in the region of the antisymmetric stretching vibration of $\mathrm{CO}_{2}$ for mixed and pure particles. Upper traces: Experimental spectra. Lower traces: Exciton calculations. Panels (a1) and (a2): Statistically mixed $\mathrm{CO}_{2}-\mathrm{NH}_{3}$ particles $\left(\mathrm{CO}_{2}: \mathrm{NH}_{3}=3: 17\right)$. Panels (b1) and (b2): Pure $\mathrm{CO}_{2}$ particles. The spectra in panels (a1) and (b1) are for predominantly globular particles. The spectra in panels (a2) and (b2) are for mostly elongated particles. See text for more details.

[Fig. 5(a) compared with Fig. 5(b)]. This statement is also true for the umbrella bands of the three other isotopomers in the mixed particles in Fig. 5: The comparison of Fig. 5(a) with Fig. 5(b) shows that none of them exhibits any dependence on the particle's shape. This result is fully confirmed by the corresponding exciton calculations which are shown in the upper traces of Fig. 5. Though the two simulations are for different particle shapes [Fig. 5(a) 1:1.7 mixture of globular and elongated particles and Fig. 5(b): 1:6 mixture of globular and elongated particles] they do not show any significant difference. This is in full agreement with the experimental spectra.

\section{B. $\mathrm{CO}_{2}-\mathrm{NH}_{3}$ mixtures}

The results found for the isotopically mixed particles are also valid for mixtures between different chemical substances. Again due to the different transition frequencies of the different substances, the resonance condition for exciton coupling is partially lifted. Therefore, also in this case, one expects at least a partial loss of the information about the particle's shape in the infrared spectra.

This is illustrated in Figs. 6 and 7 for the example of statistically mixed particles which contain $15 \% \mathrm{CO}_{2}$ and $85 \% \mathrm{NH}_{3}$. The portion of $\mathrm{CO}_{2}$ is similar to the portion of the different isotopomers in the isotopically mixed particles discussed above. Thus, we expect a total loss of information about the particle's shape in the region of the $\mathrm{CO}_{2}$ absorption in the infrared spectra of these mixed particles. Figures 6(a1) and 6(a2) show that this is indeed found experimentally as well as in the exciton calculations. The experimental and the calculated spectrum for a 1:1.7 mixture of globular and elongated particles in the region of the antisymmetric stretching vibration of $\mathrm{CO}_{2}$ is depicted in Fig. 6(a1). Figure 6(a2) shows the experimental and calculated spectrum for the same $\mathrm{CO}_{2}-\mathrm{NH}_{3}$ mixture as Fig. 6(a1) but now for predominantly elongated particles (globular:elongated $=1: 7$ ). The 

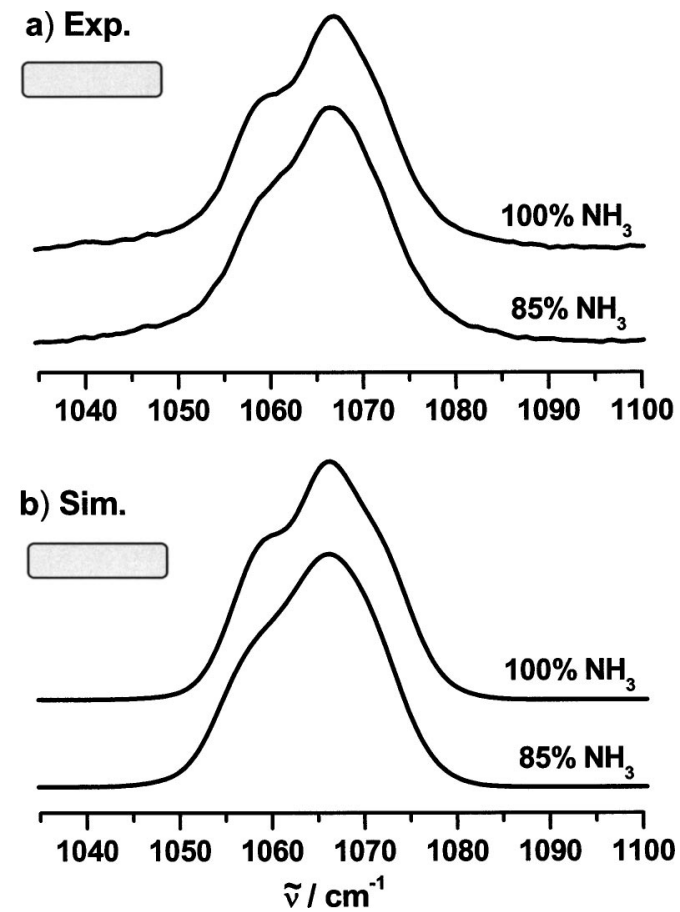

FIG. 7. (a) Infrared spectra of predominantly elongated particles in the region of the umbrella vibration of $\mathrm{NH}_{3}$. Upper trace: $\mathrm{Pure} \mathrm{NH}_{3}$ particles. Lower trace: Statistically mixed $\mathrm{CO}_{2}-\mathrm{NH}_{3}$ particles $\left(\mathrm{CO}_{2}: \mathrm{NH}_{3}=3: 17\right)$. The lower trace shows the same spectrum as Fig. 6(a2) but in a different frequency range. (b) Corresponding exciton calculations.

comparison of Fig. 6(a1) with Fig. 6(a2) demonstrates that shape effects on $\mathrm{CO}_{2}$ bands are indeed completely lost for mixed $\mathrm{CO}_{2}-\mathrm{NH}_{3}$ particles. It also illustrates that the shoulder observed on the high frequency side of the antisymmetric stretching vibration has nothing to do with shape effects as one might have supposed only from the experimental spectra alone. Our microscopic model clearly proves that this is not the case: Though the two simulations in Figs. 6(a1) and 6(a2) are for different particle shapes, they are identical.

For the two calculations shown, the particle shapes have been modelled in the same way as for the pure ammonia particles described in Sec. IV. The particles have first been build up from the crystal structure of ammonia. In a second step, we have statistically replaced $15 \%$ of the ammonia molecules by $\mathrm{CO}_{2}$ molecules as depicted in Fig. 4(c). The input parameters $\widetilde{\nu}_{M}\left(\mathrm{NH}_{3}\right)$ and $\delta \mu_{M}\left(\mathrm{NH}_{3}\right)$ for the umbrella vibration of $\mathrm{NH}_{3}$ (see below Fig. 7) are the same as given in Eq. (3). For the antisymmetric stretching vibration of $\mathrm{CO}_{2}$, we have used the following values:

$$
\tilde{\nu}_{M}\left(\mathrm{CO}_{2}\right)=2358 \mathrm{~cm}^{-1} \text { and } \delta \mu_{M}\left(\mathrm{CO}_{2}\right)=0.32 \mathrm{D}
$$

$\delta \mu_{M}\left(\mathrm{CO}_{2}\right)$ corresponds to the gas-phase value of $\mathrm{CO}_{2} \cdot{ }^{46}$ The transition frequency $\widetilde{\nu}_{M}\left(\mathrm{CO}_{2}\right)$, however, is shifted by 9 $\mathrm{cm}^{-1}$ to higher values in comparison with the corresponding gas-phase value of $2349 \mathrm{~cm}^{-1}$. This shift most probably arises from the influence of the surrounding ammonia molecules. We have again tried to verify this assumption by ab initio calculations ${ }^{48}$ for a small cluster which consists of a $\mathrm{CO}_{2}$ molecule ( $\left.\tilde{\nu}_{\text {cluster }}\right)$ surrounded by six $\mathrm{NH}_{3}$ molecules as depicted in Fig. 4(c). To estimate the influence of the surrounding $\mathrm{NH}_{3}$ molecules, the transition wavenumber $\tilde{\nu}_{\text {cluster }}$ is again compared with the transition wavenumber $\widetilde{\nu}_{\text {molecule }}$ for an isolated $\mathrm{CO}_{2}$. The corresponding shifts $\widetilde{\nu}_{\text {cluster }}$ $-\widetilde{\nu}_{\text {molecule }}$ are listed in the third column of Table II for different levels of theory. The calculations show that it is at least not unplausible that the shift of $9 \mathrm{~cm}^{-1}$ found experimentally arises from the influence of the ammonia molecules.

Figures 6(b1) and 6(b2) show that in contrast to the situation for mixed $\mathrm{CO}_{2}-\mathrm{NH}_{3}$ particles, the infrared spectra of pure $\mathrm{CO}_{2}$ particles are clearly dominated by the particle shape. Figure 6(b1) depicts an experimental and a calculated spectrum for predominantly globular particles. These spectra are dominated by the absorption of the globular particles. Figure 6(b2) depicts an experimental and a calculated spectrum for mostly elongated particles. In contrast to Fig. 6(b1), these spectra are dominated by features arising from the elongated particles.

Figure 7 illustrates the effect of mixing on the umbrella vibration of $\mathrm{NH}_{3}$. Figure 7 (a) shows experimental spectra for 1:7 mixtures of globular and elongated particles. The upper trace corresponds to pure $\mathrm{NH}_{3}$ particles. The lower trace depicts the spectrum of particles that consist of $15 \%$ $\mathrm{CO}_{2}$ and $85 \% \mathrm{NH}_{3}$ as in Fig. 6(a2). The comparison of the two traces in Fig. 7(a) reveals that the effect of mixing on the ammonia band is weaker compared with the effect on the $\mathrm{CO}_{2}$ band discussed above [Figs. 6(a2) and 6(b2)]. The lowfrequency shoulder is here only somewhat reduced in the $\mathrm{CO}_{2}-\mathrm{NH}_{3}$ particles compared with the pure $\mathrm{NH}_{3}$ particles. In other words, the mixing here leads only to a partial and not to a total loss of the information on the particle shape in the spectra. Again this corresponds exactly to the expectations. In contrast to $\mathrm{CO}_{2}$ or to the isotopomers in the isotopically mixed particles discussed in the previous subsection, $\mathrm{NH}_{3}$ represents with $85 \%$ the major component in the $\mathrm{CO}_{2}-\mathrm{NH}_{3}$ particles. Consequently, the exciton coupling is only slightly reduced in comparison with pure particles. Again, this is confirmed by the calculated spectra which are depicted in Fig. 7(b). The upper trace is for pure ammonia and the lower trace is for the $15 \% \mathrm{CO}_{2} / 85 \% \mathrm{NH}_{3}$ mixture. In agreement with the experimental spectra, the low-frequency shoulder is only slightly reduced for the mixed particles compared with the pure particles.

The results found in this section show that in general shape effects only play a minor role in the infrared spectra of mixed particles. This is in contrast to the situation found for pure particles. Figures 6 and 7 also demonstrate that a microscopic model is indispensable to understand all the various features that can occur in such complex particle spectra.

\section{SURFACE EFFECTS IN NANOSIZED PARTICLES}

The particles considered in the previous sections have sizes above about $10 \mathrm{~nm}$. In this size range, the influence of the particle's surface on the spectra is negligible compared with that of the particle's interior. This, however, is no longer true for particles smaller than about $10 \mathrm{~nm}$. For such particles, characteristic absorptions of the surface region can strongly influence the infrared spectra as has been shown in 

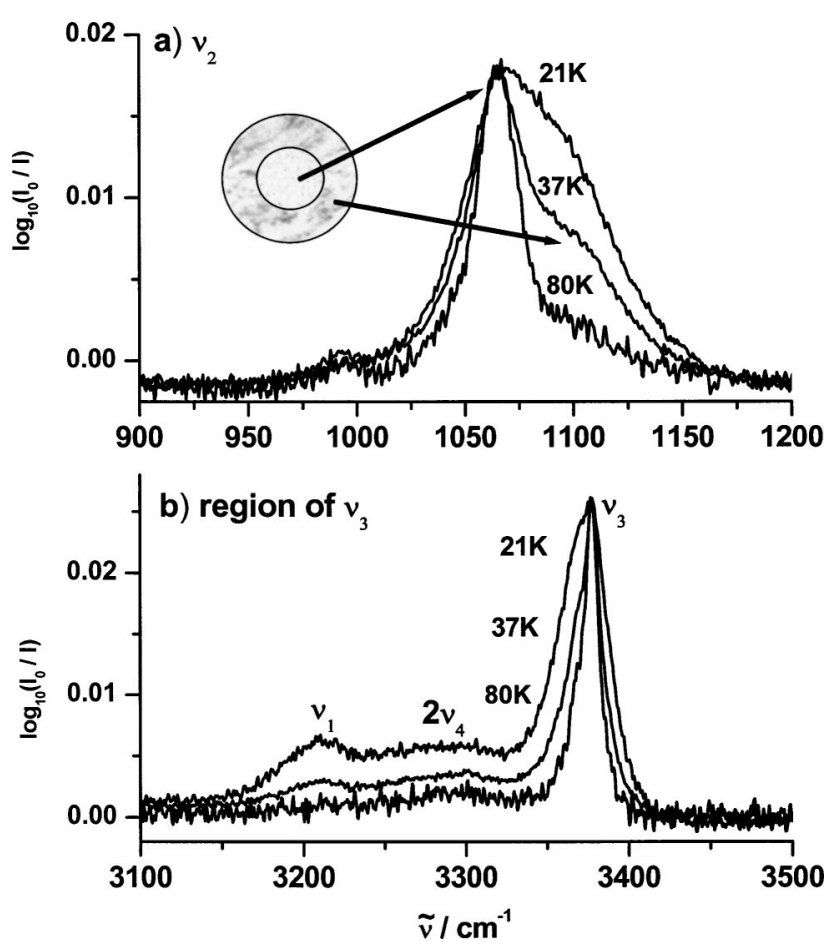

FIG. 8. Experimental infrared spectra for nanosized ammonia particles. The particles were formed from a sample gas mixture of $100 \mathrm{ppm} \mathrm{NH}_{3}$ in $\mathrm{He}$ at temperatures between 20 and $80 \mathrm{~K}$. The particle size is roughly estimated to lie around $10 \mathrm{~nm}$ for the $80 \mathrm{~K}$ spectrum, around $4 \mathrm{~nm}$ for the $37 \mathrm{~K}$ spectrum, and at $2 \mathrm{~nm}$ for the $21 \mathrm{~K}$ spectrum. (a) Region of the umbrella vibration. (b) Region of the degenerate NH-stretching mode.

previous studies on small $\mathrm{H}_{2} \mathrm{O}$ or $\mathrm{N}_{2} \mathrm{O}$ particles. ${ }^{6,8,49}$ Here, we report on the first experimental hints that similar phenomena also appear in small ammonia particles.

We have demonstrated in a previous study ${ }^{15}$ that in a collisional cooling cell small particles are generated at low bath gas temperatures (and for low sample gas concentrations). To form particles between 1 and $10 \mathrm{~nm}$, temperatures between 5 and $80 \mathrm{~K}$ are required. Such temperatures can be achieved by cooling the cell with liquid helium. Figure 8 shows infrared spectra of $\mathrm{NH}_{3}$ particles that have been recorded at three different bath gas temperatures. From a comparison with our previous study on small $\mathrm{N}_{2} \mathrm{O}$ particles, ${ }^{8}$ we can give at least a rough estimate of the corresponding particle sizes: The particle radius lies around $10 \mathrm{~nm}$ for the $80 \mathrm{~K}$ spectrum, around $4 \mathrm{~nm}$ for the $37 \mathrm{~K}$ spectrum, and at $2 \mathrm{~nm}$ for the $21 \mathrm{~K}$ spectrum. Note that this represents only a very rough estimate so that the size could easily differ by a factor of 2. The experimental conditions were chosen so that predominantly globular particles were formed.

The umbrella vibration, which is depicted in Fig. 8(a), exhibits a systematic behavior with decreasing particle size: The absorption band broadens especially on the highfrequency side. Similar size-dependent features are also observed in the region around $3300 \mathrm{~cm}^{-1}$ in Fig. 8(b) where the strongest absorption arises from the degenerate $\mathrm{NH}$ stretching mode $\nu_{3}$ around $3380 \mathrm{~cm}^{-1}$. The long wavelength tail $\left(3200-3300 \mathrm{~cm}^{-1}\right)$ is due to $\nu_{1}$ and $2 \nu_{4}$. In contrast to the umbrella band, the broadening of the stretching band is more pronounced towards the low-frequency side. For the $\nu_{3}$ band, Barth and Huisken have reported similar effects in CARS spectra of ammonia clusters. ${ }^{40}$ The radii of these clusters were estimated to lie below around $0.7 \mathrm{~nm}\left(\sim 50 \mathrm{NH}_{3}\right.$ molecules). Although these clusters are somewhat smaller than our smallest particles, a very similar spectral behavior is observed in both cases. For completeness, Table I compares the transition wavenumbers measured in the $21 \mathrm{~K}$ spectrum in Fig. 8 for $\nu_{1}, 2 \nu_{4}$, and $\nu_{3}$ with those given in Ref. 40 .

A very plausible explanation of the spectral changes observed in Fig. 8 for decreasing particle size is the occurrence of surface effects. In crystalline $\mathrm{NH}_{3}$ aggregates, the surface molecules are not completely integrated into the hydrogen bond network in contrast to the molecules in the interior of the particles. Different surroundings lead to different absorption frequencies. Our $a b$ initio calculations for the small crystalline $\left(\mathrm{NH}_{3}\right)_{7}$ cluster in Fig. 4(b) yield $\nu_{2}$ transition wavenumbers of the surrounding molecules shifted by more than $100 \mathrm{~cm}^{-1}$ relative to the wavenumber of the central molecule. This shift, however, is towards lower wavenumbers. Therefore, it does not explain the broadening in Fig. 8(a). An alternative explanation would be a surface layer structure that is different from the crystalline structure of the interior. This would also result in different absorption frequencies for these two regions. From a comparison with the calculations for small $\mathrm{NH}_{3}$ clusters ( $<18$ molecules) of Beu and Buck, ${ }^{50}$ we expect that the surface has a structure similar to such small clusters rather than to the crystalline structure of the particle's interior. According to these calculations, the clusters absorb blue shifted compared with the main absorption of the umbrella vibration around $1066 \mathrm{~cm}^{-1}$, i.e., exactly in the region where the additional absorptions in Fig. 8(a) appear. In terms of exciton coupling, this means that the resonance condition between molecules in the interior and molecules in the surface region is no longer fulfilled in these small particles. Thus, the vibrational eigenfunction is no longer delocalized over the whole particle in contrast to the situation for the large crystalline ammonia particles in Sec. IV. The same analogy between the calculations of Beu and Buck $^{50}$ and the size-dependent absorptions in our particle spectra is also found for the $\nu_{3}$ band in Fig. 8(b). Currently, we are combining vibrational dynamics calculations with molecular-dynamics simulations to find out whether these explanations are true or not. The corresponding results and comparisons with other experimental techniques to produce small particles will be the subject of a forthcoming publication.

\section{CONCLUSIONS}

The present study of ammonia ice aerosols contributes to the fundamental understanding of shape effects in the infrared spectra of molecular particles. The combination of collisional cooling and FTIR-spectroscopy with the quantum chemical exciton model explains for the first time on a molecular level why pronounced shape effects in pure particles are only observed for vibrations with strong molecular transition dipoles. It also reveals that in the case of mixed particles shape effects are in general of minor importance. This was demonstrated here for the example of isotopically mixed particles and $\mathrm{CO}_{2}-\mathrm{NH}_{3}$ mixtures. The general conclusion, 
however, is true for all kinds of mixtures. This result also has implications for the investigation of particles in the atmosphere or in interstellar dust where mixed particles often dominate. Currently, we are trying to exploit these results to improve the understanding of the particle formation in the RESS (Rapid Expansion of Supercritical Solutions) process. $^{27}$ The RESS technique is used to produce pharmaceutically active substances in the form of small particles. ${ }^{10}$

The present investigation demonstrates that collisional cooling is also a powerful method to generate particles in the nanometer size range $(1-10 \mathrm{~nm})$. We have reported on the first experimental hints that the infrared spectra of nanosized $\mathrm{NH}_{3}$ particles are governed by surface effects. The combination of these experimental results with vibrational dynamics calculations and molecular-dynamics simulations will lead to a detailed understanding of such phenomena.

\section{ACKNOWLEDGMENTS}

Financial support by the Deutsche Forschungsgemeinschaft (Grant No. SI 833/1-1, SFB 602, and GRK 782) and by the Fonds der Chemischen Industrie is gratefully acknowledged.

${ }^{1}$ M. L. Clapp and R. E. Miller, Icarus 105, 529 (1993).

${ }^{2}$ R. Disselkamp and G. E. Ewing, J. Chem. Phys. 99, 2439 (1993).

${ }^{3}$ M. L. Clapp, R. E. Miller, and D. R. Worsnop, J. Phys. Chem. 99, 6317 (1995).

${ }^{4}$ D. D. Weis and G. E. Ewing, J. Geophys. Res. 101, 18709 (1996).

${ }^{5}$ T. E. Gough and T. Wang, J. Chem. Phys. 105, 4899 (1996).

${ }^{6}$ J. P. Devlin, C. Joyce, and V. Buch, J. Phys. Chem. A 104, 1974 (2000).

${ }^{7}$ J. P. Devlin, N. Uras, J. Sadlej, and V. Buch, Nature (London) 417, 269 (2002).

${ }^{8}$ M. K. Kunzmann, S. Bauerecker, M. A. Suhm, and R. Signorell, Spectrochim. Acta, Part A 59, 2855 (2003).

${ }^{9}$ R. Signorell and M. K. Kunzmann, Chem. Phys. Lett. 371, 260 (2003).

${ }^{10}$ M. Türk, B. Helfgen, P. Hils, R. Lietzow, and K. Schaber, Part. Part. Syst. Charact. 19, 327 (2002)

${ }^{11}$ J. H. Seinfeld and S. N. Pandis, Atmospheric Chemistry and Physics (Wiley, New York, 1998).

${ }^{12}$ P. Ehrenfreund and S. B. Charnley, Annu. Rev. Astron. Astrophys. 38, 427 (2000).

${ }^{13}$ C. F. Bohren and D. R. Huffman, Absorption and Scattering of Light by Small Particles (Wiley-Interscience, New York, 1998).

${ }^{14}$ M. I. Mishchenko, J. W. Hovenier, and L. D. Travis, Light Scattering by Nonspherical Particles (Academic, San Diego, 2000).

${ }^{15}$ M. K. Kunzmann, R. Signorell, M. Taraschewski, and S. Bauerecker, Phys. Chem. Chem. Phys. 3, 3742 (2001).
${ }^{16}$ S. Bauerecker, M. Taraschewski, C. Weitkamp, and H. K. Cammenga, Rev. Sci. Instrum. 72, 3946 (2001).

${ }^{17}$ R. Signorell and D. Luckhaus, J. Phys. Chem. A 106, 4855 (2002).

${ }^{18}$ R. M. Hexter, J. Chem. Phys. 33, 1833 (1960).

${ }^{19}$ G. Cardini, V. Schettino, and M. L. Klein, J. Chem. Phys. 90, 4441 (1989).

${ }^{20}$ R. Disselkamp and G. E. Ewing, J. Chem. Soc., Faraday Trans. 86, 2369 (1990).

${ }^{21}$ D. J. Wales and G. E. Ewing, J. Chem. Soc., Faraday Trans. 88, 1359 (1992).

${ }^{22}$ M. A. Ovchinnikov and C. A. Wight, J. Chem. Phys. 100, 972 (1994).

${ }^{23}$ R. Signorell, J. Chem. Phys. 118, 2707 (2003).

${ }^{24}$ A. Bonnamy, M. Jetzki, and R. Signorell, Chem. Phys. Lett. 382, 547 (2003).

${ }^{25}$ Th. Koops, T. Visser, and W. M. A. Smit, J. Mol. Struct. 96, 203 (1983).

${ }^{26}$ B. J. Krohn and L. H. Jones, J. Mol. Spectrosc. 86, 16 (1981).

${ }^{27}$ R. Signorell, Mol. Phys. 101, 3385 (2003).

${ }^{28}$ J. V. Martonchik, G. S. Orton, and J. F. Appleby, Appl. Opt. 23, 541 (1984).

${ }^{29}$ J. A. Roux, B. E. Wood, and A. M. Smith, IR optical properties of thin $\mathrm{H}_{2} \mathrm{O}, \mathrm{NH}_{3}$, and $\mathrm{CO}_{2}$ cryofilms. AEDC-TR-79-57 (AD-A074913), September, 1979.

${ }^{30}$ F. P. Reding and D. F. Hornig, J. Chem. Phys. 19, 594 (1951).

${ }^{31}$ F. P. Reding and D. F. Hornig, J. Chem. Phys. 23, 1053 (1955).

${ }^{32}$ H. Wolff, H.-G. Rollar, and E. Wolff, J. Chem. Phys. 55, 1373 (1971).

${ }^{33}$ A. Bromberg, S. Kimel, and A. Ron, Chem. Phys. Lett. 46, 262 (1977).

${ }^{34}$ A. D. Fortes, J. P. Brodholt, I. G. Wood, and L. Vočadlo, J. Chem. Phys. 118, 5987 (2003).

${ }^{35}$ R. Boese, N. Niederprüm, D. Bläser, A. Maulitz, M. Y. Antipin, and P. R. Mallinson, J. Phys. Chem. B 101, 5794 (1997).

${ }^{36}$ A. W. Hewat and C. Riekel, Acta Crystallogr., Sect. A: Cryst. Phys., Diffr., Theor. Gen. Crystallogr. 35, 569 (1979).

${ }^{37}$ J. W. Reed and P. M. Harris, J. Chem. Phys. 35, 1730 (1961).

${ }^{38}$ I. Olovsson and D. H. Templeton, Acta Crystallogr. 12, 832 (1959).

${ }^{39}$ J. Huang and L. S. Bartell, J. Phys. Chem. 98, 4543 (1994).

${ }^{40}$ H. D. Barth and F. Huisken, J. Chem. Phys. 87, 2549 (1987).

${ }^{41}$ J. A. Barnes, T. E. Gough, and M. Stoer, J. Chem. Phys. 95, 4840 (1991).

${ }^{42}$ S. K. Pope, M. G. Tomasko, M. S. Williams, M. L. Perry, L. R. Doose, and P. H. Smith, Icarus 100, 203 (1992).

${ }^{43}$ R. A. West, G. S. Orton, B. T. Draine, and E. A. Hubbell, Icarus 80, 220 (1989).

${ }^{44}$ B. Galabov, T. Dudev, and S. Ilieva, Spectrochim. Acta, Part A 51, 739 (1995).

${ }^{45}$ A. J. van Straten and W. M. A. Smit, J. Mol. Spectrosc. 65, 202 (1977).

${ }^{46}$ L. S. Rothman and W. S. Benedict, Appl. Opt. 17, 2605 (1978).

${ }^{47}$ T. Rajamäki, A. Miani, and L. Halonen, J. Chem. Phys. 118, 6358 (2003).

${ }^{48}$ M. J. Frisch, G. W. Trucks, H. B. Schlegel et al., GAUSSIAN 98, Revision A7, Gaussian, Inc., Pittsburgh, PA, 1998.

${ }^{49}$ Th. Häber, U. Schmitt, C. Emmeluth, and M. A. Suhm, Faraday Discuss. 118, 331 (2001).

${ }^{50}$ T. A. Beu and U. Buck, J. Chem. Phys. 114, 7853 (2001). 